\title{
Sinais e sintomas de doença da superfície ocular em usuários de hipotensores oculares tópicos
}

\author{
Signs and symptoms of ocular surface disease in patients on topical intraocular \\ pressure-lowering therapy
}

Beatriz Gomes ${ }^{1,2}$, Paulo Romeu de Freitas Turiel ${ }^{2}$, Fabiane Pereira Marques², Fernanda Pessoa Bernardo², Marcus Vinicius Abbud Safady², André Luís Freire Portes², Marcony Rodrigues Santhiago ${ }^{1}$

\section{RESUMO}

Objetivo: determinar a prevalência de sinais e sintomas de doença da superfície ocular (OSD) em pacientes em uso crônico de hipotensores oculares tópicos.

Métodos: Neste estudo transversal, foram recrutados 40 pacientes consecutivos, provenientes do ambulatório de glaucoma de um hospital público localizado no Rio de Janeiro, Brasil. Os mesmos deveriam apresentar: idade maior ou igual a 18 anos, diagnóstico de hipertensão ocular ou glaucoma primário de ângulo aberto e deveriam estar em uso da mesma terapia hipotensora ocular há pelo menos seis meses. Foram considerados: sexo, idade, medicação utilizada e duração do tratamento. Todos os pacientes foram submetidos à avaliação da superfície ocular que incluiu: entrevista por meio do questionário Ocular Surface Disease Index ${ }^{\circledR}$ $\left(\mathrm{OSD} I^{\circledR}\right)$, tempo de rotura do filme lacrimal, biomicroscopia, avaliação da superfície ocular com fluoresceína e com rosa Bengala.

Resultados: A média de pontuação do OSDI ${ }^{\circledR}$ foi $24,6 \pm 20,7$. A maioria dos pacientes $(67,5 \%)$ apresentou uma pontuação anormal no questionário do OSD ${ }^{\circledR}$. Em $25 \%$ dos pacientes, a pontuação foi compatível com sintomas leves, em 12,5\% com sintomas moderados e em 30\% com sintomas graves. Blefarite e ceratite ponteada foram diagnosticadas em $42,5 \%$ e $20 \%$ dos pacientes respectivamente. Instabilidade do filme lacrimal foi observada em 75\% dos pacientes, enquanto que alteração da superfície ocular foi evidenciada pelo teste de rosa bengala em $35 \%$ dos pacientes. Foi encontrada correlação positiva $(r=0,4)$ estatisticamente significativa $(p=0,01)$ entre a pontuação do OSDI ${ }^{\circledR}$ e o tempo de duração do tratamento com hipotensores oculares tópicos. Conclusão: Pacientes em uso crônico de hipotensores oculares tópicos apresentam alta prevalência de sinais e sintomas de OSD. Existe correlação significativa entre a duração do tratamento e a gravidade dos sintomas de OSD.

Descritores: Glaucoma de ângulo aberto; Hipertensão ocular; Ceratoconjuntivite seca; Blefarite; Compostos de benzalcônio

\begin{abstract}
Purpose: To determine the prevalence of signs and symptoms ofocular surface disease (OSD) in patients using topical intraocular pressure-lowering therapy.

Methods: In this cross-sectional study, 40 patients were consecutively recruited from the glaucoma clinic of a public hospital located in Rio de Janeiro, Brazil. Eligible patients were 18 years of age or older, with primary open-angle glaucoma or ocular hypertension and on the same topical ocular therapy for at least 6 months. The following data were considered: sex, age, medication history and number of years on topical intraocular pressure-lowering therapy. All patients underwent an evaluation of the ocular surface which included: an interview using the Ocular Surface Disease Index ${ }^{\circledR}$ $\left(O S D{ }^{\circledR}\right)$ questionnaire, break-up time, biomicroscopy, fluorescein corneal staining and rose Bengal ocular surface staining.

Results: The mean $O S D{ }^{\circledR}$ score was $24.6 \pm 20.7$. Most patients (67.5\%) had an abnormal score on the $\mathrm{OSD}^{\circledR}{ }^{\circledR}$ questionnaire. In $25 \%$ of patients, the score was consistent with mild symptoms, $12.5 \%$ with moderate symptoms and $30 \%$ with severe symptoms. Blepharitis and punctate keratitis were diagnosed in $42.5 \%$ and $20 \%$ of patients respectively. Tear film instability was observed in $75 \%$ of patients and ocular surface staining with rose Bengal in 35\%. A positive statistically significant correlation ( $r=0.4$; $p=0.01$ ) was found between OSD ${ }^{\circledR}$ scores and the duration of topical intraocular pressure-lowering therapy.
\end{abstract}

Conclusion: Patients with primary open-angle glaucoma or ocular hypertension on topical intraocular pressure-lowering therapy have high prevalence of OSD. Longer duration since diagnosis is significantly correlated with worsening of OSD symptoms.

Keywords: Open-angle glaucoma; Ocular hypertension; Keratoconjunctivitis sicca; Blepharitis; Benzalkonium compounds

\section{INTRODUÇÃO}

A doença da superfície ocular (OSD) representa uma das alterações mais prevalentes no ambulatório de oftalmologia(1). Manifesta-se principalmente como condições alérgicas, tóxicas ou pró-inflamatórias, provocando sintomas como ressecamento ocular, irritação, queimação, lacrimejamento, sensação de corpo estranho, olho vermelho e visão turva(1,2).

O surgimento da OSD é um fenômeno multifatorial, sendo influenciado pela idade, etnia e sexo do indivíduo e por fatores ambientais, podendo estar associado a outras desordens oftalmológicas como disfunção das glândulas meibomianas e blefarite ${ }^{(2)}$. Além disso, medicações de uso sistêmico e tópico podem contribuir para o surgimento de OSD ${ }^{(2-5)}$.

A prevalência de OSD na população geral varia de forma significativa (0,39 a 33,7\%), sendo maior com a idade e no sexo feminino(2). Esta variação pode ser explicada pela diversidade dos critérios diagnósticos adotados pelos diferentes estudos e pelas diferentes populações e áreas geográficas estudadas ${ }^{(2)}$.
Submetido para publicação: 22 de maio de 2012

Aceito para publicação: 29 de maio de 2013

Trabalho realizado no Serviço de Oftalmologia do Hospital Federal de Bonsucesso, Rio de Janeiro (RJ), Brasil.

Médico, Universidade Federal do Rio de Janeiro, Rio de Janeiro (RJ), Brasil.

${ }^{2}$ Médico, Hospital Federal de Bonsucesso - Rio de Janeiro (RJ), Brasil.
Financiamento: Não houve financiamento para este trabalho.

Divulgação de potenciais Conflitos de Interesse: B.Gomes, Nenhum; P.R.F.Turiel, Nenhum; F.P.Marques, Nenhum; F.P.Bernardo, Nenhum; M.V.A.Safady, Nenhum; A.L.F.Portes, Nenhum M.R.Santhiago, Nenhum.

Endereço de correspondência: Beatriz Gomes. Rua Real Grandeza, 139 sala 301 - Rio de Janeiro (RJ) - 22281-033 - E-mail: exopina@hotmail.com

Aprovação no Comitê de ética e Pesquisa do Hospital Federal de Bonsucesso sob o número CEP-HFB 50/11. 
Pacientes com hipertensão ocular $(\mathrm{OHT})$ e pacientes com glaucoma primário de ângulo aberto (GPAA) apresentam maior probabilidade de desenvolver danos à superfície ocular, se comparados à população normal(4,5). Sabe-se que tanto o próprio princípio ativo dos colírios hipotensores oculares, quanto o conservante utilizado, geralmente o cloreto de benzalcônio (BAK), podem provocar e/ou agravar alterações na superfície ocular ${ }^{(4,6)}$.

Estudos recentes tem demonstrado alta prevalência de sinais e sintomas de OSD em pacientes com glaucoma em diversas áreas geográficas ${ }^{(3,5,7,8)}$. Contudo, este assunto foi pouco estudado na América Latina e a prevalência no Brasil ainda é desconhecida ${ }^{(7)}$.

A presença de OSD em pacientes com glaucoma afeta a qualidade de vida dos pacientes e é possível que tenha influência na aderência terapêutica ${ }^{(8-10)}$. Além disso, sabe-se que a superfície ocular saudável é importante para o sucesso da trabeculectomia(11). Sendo assim, é fundamental conhecer a prevalência da OSD em pacientes com glaucoma/hipertensão ocular em uso crônico de hipotensores oculares tópicos, bem como os principais fatores associados a estas patologias, a fim de proporcionar aos mesmos melhor manejo terapêutico e qualidade de vida.

\section{Objetivo}

Determinar a prevalência de sinais e sintomas de OSD em pacientes em uso crônico de hipotensores oculares tópicos.

\section{MÉTODOS}

Com a aprovação do comitê de ética em pesquisa do Hospital Federal de Bonsucesso (HFB), foi realizado estudo transversal, visando determinar a prevalência dos sinais e sintomas de OSD em pacientes em uso crônico de hipotensores oculares tópicos.
Os pacientes foram recrutados, de forma consecutiva, do ambulatório de glaucoma do HFB (Rio de Janeiro, Brasil) durante período de dois meses. Como critérios de inclusão, os pacientes deveriam apresentar: idade maior ou igual a 18 anos, diagnóstico de OHT ou GPAA e deveriam estar em uso da mesma terapia hipotensora ocular tópica há pelo menos seis meses. Foram adotados como critérios de exclusão: (1) uso de Restasis ${ }^{\circledR}$, corticosteroide tópico ou anti-inflamatório não esteroide tópico nos últimos três meses; (2) uso de colírio lubrificante ocular nos últimos sete dias; (3) uso atual de plug lacrimal; (4) cirurgia ocular nos últimos 12 meses; (5) doença crônica sistêmica (diabetes melitus, artrite reumatoide) ou ocular (rosácea, distrofias corneanas); (6) alteração estrutural das pálpebras (ectrópio, entrópio, triquíase).

Os seguintes dados foram considerados: sexo, idade, medicação utilizada, duração do tratamento com colírios hipotensores oculares. Todos os pacientes foram submetidos à avaliação cuidadosa da superfície ocular que incluiu: entrevista por meio do questionário Ocular Surface Disease Index ${ }^{\circledR}\left(\mathrm{OSDl}{ }^{\circledR}\right)$, tempo de rotura do filme lacrimal (TRFL), biomicroscopia, avaliação da superfície corneana com fluoresceína e teste de coloração da superfície ocular com rosa Bengala.

Para avaliação dos sintomas todos os pacientes foram submetidos questionário "Ocular Surface Disease Index ${ }^{\circledR "}(\mathrm{OSD})^{\circledR}$, Allergan, Irvine, CA, EUA)(12) (Quadro 1). O questionário foi aplicado de forma individual pelo mesmo investigador ao longo do estudo que desconhecia os dados clínicos dos pacientes bem como os objetivos deste estudo. As questões do OSDI cobrem três áreas: sintomas oculares nos sete dias anteriores à pesquisa, limitações nas atividades diárias dos pacientes em função do quadro clínico ocular e finalmente, possíveis estímulos ambientais causadores de desconforto ocular. Ao final, um índice é gerado, levando em consideração tanto os escores fornecidos em cada uma das respostas como o número total de perguntas que puderam ser respondidas. O índice do OSDI ${ }^{\circledR}$

Quadro 1. Questionário Ocular Surface Disease Index ${ }^{\circledR}$

\section{Avaliação OSDI ${ }^{\odot}$}

Responda as 12 perguntas a seguir marcando com um X a alternativa que melhor represente cada resposta. Posteriormente, entregue o questionário a seu médico oftalmologista.

Você experimentou qualquer dos seguintes sintomas durante a ultima semana?

\begin{tabular}{|c|c|c|c|c|c|}
\hline & $\begin{array}{l}\text { O tempo } \\
\text { todo }\end{array}$ & $\begin{array}{l}\text { A maior parte } \\
\text { do tempo }\end{array}$ & $\begin{array}{l}\text { A metade } \\
\text { do tempo }\end{array}$ & Às vezes & Nunca \\
\hline 1. Olhos sensíveis à luz? & $\square$ & $\square$ & $\square$ & $\square$ & $\square$ \\
\hline 2. Sensação de areia nos olhos? & $\square$ & $\square$ & $\square$ & $\square$ & $\square$ \\
\hline 3. Dor ou vermelhidão dos olhos? & $\square$ & $\square$ & $\square$ & $\square$ & $\square$ \\
\hline 4. Visão embaçada? & $\square$ & $\square$ & $\square$ & $\square$ & $\square$ \\
\hline 5. Visão deficiente? & $\square$ & $\square$ & $\square$ & $\square$ & $\square$ \\
\hline
\end{tabular}

Os problemas com os seus olhos límitou você na realização de alguma das seguintes atividades durante a última semana?

\begin{tabular}{|c|c|c|c|c|c|}
\hline & $\begin{array}{l}\text { O tempo } \\
\text { todo }\end{array}$ & $\begin{array}{l}\text { A maior parte } \\
\text { do tempo }\end{array}$ & $\begin{array}{l}\text { A metade } \\
\text { do tempo }\end{array}$ & Às vezes & Nunca \\
\hline 6. Ler? & $\square$ & $\square$ & $\square$ & $\square$ & $\square$ \\
\hline 7. Dirigir à noite? & $\square$ & $\square$ & $\square$ & $\square$ & $\square$ \\
\hline 8. Trabalhar com o computador ou usar o caixa eletrônico? & $\square$ & $\square$ & $\square$ & $\square$ & $\square$ \\
\hline 9. Assistir televisão? & $\square$ & $\square$ & $\square$ & $\square$ & $\square$ \\
\hline \multicolumn{6}{|c|}{ Você sentiu algum desconforto nos seus olhos em alguma das seguintes situações durante a última semana? } \\
\hline & $\begin{array}{l}\text { O tempo } \\
\text { todo }\end{array}$ & $\begin{array}{c}\text { A maior parte } \\
\text { do tempo }\end{array}$ & $\begin{array}{l}\text { A metade } \\
\text { do tempo }\end{array}$ & Às vezes & Nunca \\
\hline 10. Com vento? & $\square$ & $\square$ & $\square$ & $\square$ & $\square$ \\
\hline 11. Em locais ou áreas muito secas? & $\square$ & $\square$ & $\square$ & $\square$ & $\square$ \\
\hline 12. Locais com ar condicionado? & $\square$ & $\square$ & $\square$ & $\square$ & $\square$ \\
\hline
\end{tabular}


é calculado com a seguinte fórmula: OSD ${ }^{\circledR}=[$ (soma dos escores para todas as perguntas respondidas) $\times 100] /$ [(total de perguntas respondidas) $x 4]$. Os valores possíveis variam de zero a 100, sendo que quanto maior o índice, maior o impacto dos sintomas na qualidade de vida dos pacientes ${ }^{(13)}$. Para interpretação dos resultados, uma pontuação de 0 a 12 foi classificada como normal, 13 a 22 como sintomatologia leve, 23 a 32 como moderada, e 33 a 100 como grave co,13). $^{(2)}$.

Os testes clínicos de análise da superfície ocular foram realizados por investigador mascarado para os resultados do questionário OSD ${ }^{\circledR}$ e perfil medicamentoso dos pacientes. A avaliação do TRFL foi realizada instilando-se uma gota de fluoresceína sódica a 1\% (Fluoresceína ${ }^{\circledR}$, Allergan, São Paulo, SP, Brasil) no fundo de saco inferior. O examinador, utilizando luz de cobalto na lâmpada de fenda, determinou, com o uso de um cronômetro, o tempo em segundos da abertura do olho até o aparecimento da primeira mancha seca. Valores menores que 10 segundos foram considerados anormais. Os resultados anormais foram classificados como "leve a moderado" se entre 5 e 9 e "grave" se menor que 5 segundos(3). Em seguida, foi realizada a avaliação da superfície corneana pela coloração com fluoresceína e a presença de alteração do padrão normal foi registrada. A avaliação da superfície ocular após coloração com rosa Bengala foi realizada através do uso de uma fita impregnada por rosa Bengala (Rose Glo ${ }^{\circledR}$, Rose Stone Enterprises, Alta Loma, CA, EUA) molhada com solução salina não preservada. Não foi utilizado colírio anestésico. O resultado foi graduado de acordo com o método de Van Bijsterveld ${ }^{(14)}$ e foi descrito como "leve a moderado" se menor que 3 e "grave" se maior ou igual a 3 .

Os dados deste estudo foram inseridos em um banco de dados e processados através do programa estatístico JMP, versão 8.0 (SAS Institute Inc., Cary, NC, EUA). Variáveis numéricas foram expressas pela média \pm desvio-padrão (mínimo-máximo), enquanto que variáveis categóricas foram expressas como frequência e porcentagem (\%). Os pacientes foram divididos em dois grupos de acordo com a posologia: grupo 1 (1 ou 2 instilações/ dia) e grupo 2 (3 ou mais instilações/dia). Para comparações de variáveis contínuas foi utilizado o teste de Wilcoxon-Mann-Whitney test. Para comparações de variáveis categóricas foi aplicado o teste exato de Fisher. Foi realizada regressão linear para estudar a relação entre a pontuação do OSDI e a duração do tratamento com hipotensores oculares. Um p valor $<0,05$ foi considerado estatisticamente significativo.

\section{RESULTADOS}

Um total de 40 pacientes (80 olhos) foram incluídos neste estudo. A média de idade foi $60,8 \pm 13,9$ (24-87) anos. Os pacientes apresentaram um tempo médio de tratamento com hipotensores oculares de 9,8 \pm 5,9 (1-26) anos. Todos os pacientes estavam em uso de colírios que continham BAK como conservante. As características demográficas da amostra estão contidas na tabela 1. Os hipotensores oculares utilizados pelos pacientes estão listados na tabela 2. Dos 30 pacientes em uso de mais de um principio ativo, 9 (30\%) estavam em uso de alguma combinação fixa.

A tabela 3 apresenta o número (\%) de pacientes conforme gravidade dos resultados obtidos com os testes: tempo de rotura do filme lacrimal e coloração com rosa Bengala. A média de pontuação do OSDI ${ }^{\circledR}$ foi 24,6 $\pm 20,7(0-68,8)$. A maioria dos pacientes $(67,5 \%)$ apresentou uma pontuação anormal no questionário do OSDI ${ }^{\circledR}$. Em 25\% dos pacientes deste estudo a pontuação foi compatível com sintomas leves, em 12,5\% com sintomas moderados e em 30\% com sintomas graves (Gráfico 1).

Blefarite e ceratite ponteada foram diagnosticadas em 42,5\% e 20\% dos pacientes respectivamente. Instabilidade do filme lacrimal avaliada através do TRFL foi observada em $75 \%$ dos pacientes. A média do TRFL foi 4,9 \pm 3,3 (1-15) segundos. Em relação à avaliação com corante de rosa Bengala, 35\% dos pacientes apresentaram alguma coloração da superfície ocular em pelo menos um olho, sendo que apenas dois pacientes apresentaram uma pontuação igual ou maior
Tabela 1. Características da população do estudo

\begin{tabular}{lc}
\hline \multicolumn{2}{c}{ Características demográficas } \\
\hline Variáveis & População do estudo \\
\hline Média de idade (anos) & 60,8 \\
Desvio-padrão & 13,9 \\
Sexo, no (\%) & $32(80 \%)$ \\
Feminino & $8(20 \%)$ \\
Masculino & \\
Grau de escolaridade, no (\%) & $14(35,0 \%)$ \\
Fundamental incompleto & $15(37,5 \%)$ \\
Fundamental completo & $11(27,5 \%)$ \\
Ensino médio & 9,8 \\
Duração média do tratamento (anos) & 5,9 \\
Desvio-padrão & \\
Posologia, no (\%) & $10(25 \%)$ \\
Até 2 gotas/ dia & $30(75 \%)$ \\
3 ou mais gotas/ dia & \\
Concentração de BAK* no (\%) & $23(57,5 \%)$ \\
$<0,01$ & $17(42,5 \%)$ \\
$>0,01$ & \\
\hline BAK= co &
\end{tabular}

BAK= cloreto de benzalcônio.

* = referente à medicação utilizada pelo paciente com maior concentração de BAK, nos casos de uso de múltiplos colírios.

Tabela 2. Princípio ativo dos hipotensores oculares utilizados pelo grupo estudado

\begin{tabular}{lc}
\hline \multicolumn{2}{c}{ Princípio ativo das medicações utilizadas } \\
\hline Medicação & $\mathbf{n}(\%)$ \\
\hline Maleato de timolol 0,5\% & $7(17,5 \%)$ \\
Cloridrato de dorzolamida 2\% & $1(2,5 \%)$ \\
Bimatoprosta 0,03\% & $1(2,5 \%)$ \\
Tartarato de brimonidina 0,2\% & $1(2,5 \%)$ \\
Maleato de timolol 0,5\% e cloridrato de dorzolamida 2\% & $9(22,5 \%)$ \\
Maleato de timolol 0,5\% e tartarato de brimonidina 0,2\% & $5(12,5 \%)$ \\
Maleato de timolol 0,5\% e bimatoprosta 0,03\% & $3(7,5 \%)$ \\
Maleato de timolol 0,5\% e travoprosta 0,004\% & $3(7,5 \%)$ \\
Travoprosta 0,004\% e tartarato de brimonidina 0,2\% & $2(5,0 \%)$ \\
Maleato de timolol 0,5\% e bimatoprosta 0,03\% e & $2(5,0 \%)$ \\
cloridrato de dorzolamida 2\% & \\
Maleato de timolol 0,5\% e travoprosta 0,004\% e & $2(5,0 \%)$ \\
tartarato de brimonidina 0,2\% & \\
Maleato de timolol 0,5\% e cloridrato de dorzolamida 2\% e \\
travoprosta 0,004\% e tartarato de brimonidina 0,2\%
\end{tabular}

que três, segundo a escala de Van Bijsterveld. A média da pontuação obtida com o teste de rosa Bengala foi 0,5 \pm 1(0 -5).

Não foi encontrada diferença estatisticamente significativa entre os grupos quanto ao sexo, idade, duração do tratamento, pontuação do OSDI, TRFL e teste com rosa Bengala (Tabela 4). Foi encontrada correlação positiva $(r=0,4)$ estatisticamente significativa $(p=0,01)$ entre a pontuação do $O S D{ }^{\circledR}$ e o número de anos de terapia com hipotensores oculares (Gráfico 2). 
Tabela 3. Distribuição conforme a gravidade dos resultados obtidos com o tempo de rotura do filme lacrimal e com o teste com rosa Bengala

\begin{tabular}{lcr}
\hline Resultado & TRFL & RB \\
\hline Normal & $7(17,5 \%)$ & $26(65 \%)$ \\
Leve a moderado & $23(57,5 \%)$ & $12(30 \%)$ \\
Grave & $10(25,0 \%)$ & $2(5 \%)$ \\
\hline
\end{tabular}

$\mathrm{TRF} L=$ tempo de rotura do filme lacrimal; $\mathrm{RB}=$ teste com rosa Bengala.

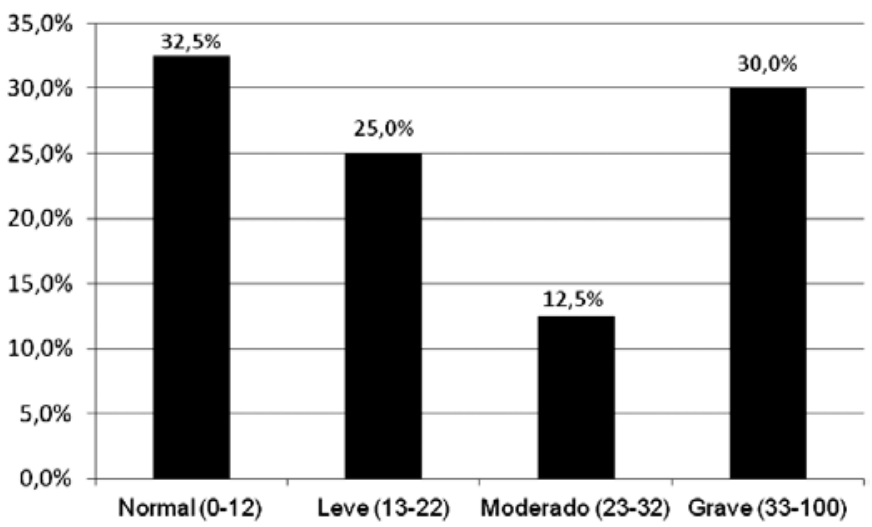

Gráfico 1. Distribuição conforme a gravidade da pontuação obtida com o questionário Ocular Surface Disease Index ${ }^{\circledR}\left(\mathrm{OSDI}^{\circledR}\right)$

\section{DISCUSSÃO}

O presente estudo demonstrou alta prevalência de OSD em pacientes com GPAA ou OHT em uso de hipotensores oculares tópicos. A OSD costuma acometer pacientes da mesma faixa etária que o glaucoma ${ }^{(2,3)}$. Neste estudo, a maioria dos pacientes encontrava-se entre a quinta e sétima década de vida, dados muito semelhantes aos encontrados em um estudo prévio realizado em hospital universitário, onde a média de idade encontrava-se em 64,1 $\pm 12,9^{(15)}$. O binômio olho seco - glaucoma é ratificado em um estudo realizado pela Universidade de Minnesota, no qual dos 108 olhos com OSD avaliados, 71 foram diagnosticados como glaucomatosos ${ }^{(16)}$.

Sabe-se que colírios hipotensores oculares podem induzir alterações na superfície ocular, tanto pelo princípio ativo propriamente dito quanto pelo conservante ${ }^{(4,16-19)}$. Neste estudo, a maior parte dos indivíduos fazia uso de medicação tópica três ou mais vezes por dia (75\%) e um número grande de pacientes (43\%) utilizava pelo menos uma medicação com concentração de BAK maior que 0,01.

Fechtner et al, em um estudo multicêntrico com 630 pacientes com glaucoma, diagnosticaram 48,4\% com OSD, e nestes, maior tendência à sintomatologia mais severa (maior pontuação no questionário OSDI ${ }^{\circledR}$ ) naqueles que utilizavam terapia múltipla, se comparados àqueles que faziam monoterapia (score OSDI ${ }^{\circledR}$ de 12,9 $\pm 13,1$; $16,7 \pm 17,0$ e 19,4 \pm 18,1 para aqueles que utilizavam 1, 2 e 3 drogas, respectivamente) ${ }^{(10)}$. Avaliando a toxicidade ocular dos preservativos existentes nos colírios antiglaucomatosos, através de um estudo prospectivo, Pisella et al ${ }^{(20)}$, mostraram a significativa prevalência dos sintomas de OSD naqueles que utilizavam terapia hipotensora que continha conservantes, se comparados aos livres do mesmo: sensação de queimação (40\% contra 22\%), sensação de corpo estranho (31\% contra 14\%), lacrimejamento (21\% contra $14 \%$ ) e prurido ocular (18\% contra 10\%). Também se observou que os sinais e sintomas eram dose-dependente, com aumento proporcional ao número de gotas antiglaucomatosas/dia ${ }^{(20)}$. Isto pode explicar em parte a
Tabela 4. Comparação entre pacientes que instilam 1 a 2 gotas de colírio hipotensor ocular/dia (grupo 1) com pacientes em uso de 3 ou mais gotas/dia (grupo 2)

\begin{tabular}{lccc}
\hline Variável & $\begin{array}{c}\text { Grupo 1 } \\
\text { N=10 }\end{array}$ & $\begin{array}{c}\text { Grupo 2 } \\
\mathbf{N = 3 0}\end{array}$ & P valor \\
\hline Idade (anos) & 57,3 & 61,9 & $0,52^{+}$ \\
Sexo feminino n (\%) & $7(70 \%)$ & $25(83 \%)$ & $0,39^{*}$ \\
Duração do tratamento (anos) & 9,4 & 9,9 & $0,99^{+}$ \\
OSDI (pontuação) & 36,1 & 20,7 & $0,11^{+}$ \\
TRFL (segundos) & 7 & 7,6 & $0,82^{+}$ \\
Teste de RB (pontuação) & 0,4 & 0,7 & $0,98^{+}$ \\
\hline
\end{tabular}

Grupo 1 = 1 ou 2 gotas/dia; Grupo 2= 3 ou mais gotas/dia; OSDI= Ocular Surface Disease Index; TRFL = tempo de rotura do filme lacrimal; $\mathrm{RB}=$ rosa Bengala.

* = teste extato de Fisher.

${ }^{+}=$Wilcoxon-Mann-Whitney test.

Não foi encontrada diferença estatisticamente significativa entre os grupos para as variáveis estudadas.

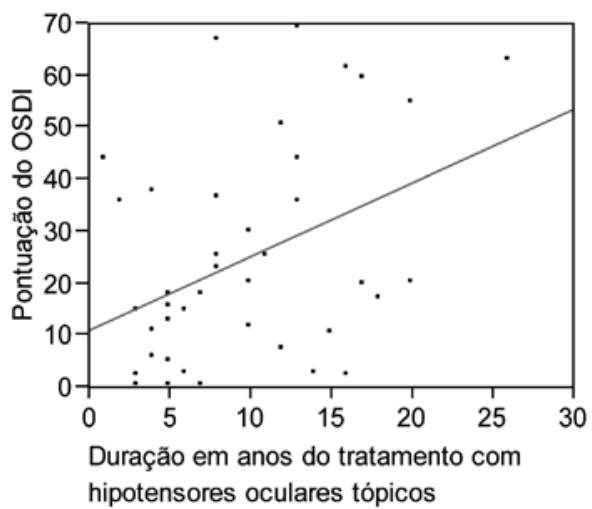

Gráfico 2: Correlação entre pontuação do questionário Ocular Surface Disease Index ${ }^{\circledR}$ $(\mathrm{OSDI})^{\circledR}$ ) e duração (número de anos) do tratamento com hipotensores oculares tópicos (Coeficiente de Pearson=0,4; $\mathrm{p}=0,01$ ).

alta pontuação média no questionário OSDI ${ }^{\circledR}$ obtida neste estudo $(24,6 \pm 20,7)$, bem como a prevalência de $42,5 \%$ dos pacientes apresentando sintomas moderados a severos.

A área geográfica estudada também pode ter contribuído para a maior gravidade dos sintomas no presente estudo. Garcia-Feijoo e Sampaolesi, ${ }^{(7)}$ em estudo multicêntrico internacional, encontararm uma diferença estatisticamente significativa na pontuação média do OSDI ${ }^{\circledR}$ em pacientes com glaucoma sul-americanos $(29 \pm 21)$ quando comparados com asiáticos (17 $\pm 17, \mathrm{p}=0.0001)$ e entre sulamericanos quando comparados com caucasianos ( $20 \pm 17, p=0.0009)$. Pacientes sul-americanos portadores de glaucoma apresentaram, portanto, uma pontuação média no OSDI ${ }^{\circledR}$ compatível com sintomas moderados, e similar à encontrada no estudo atual. Já a pontuação média do OSDI ${ }^{\circledR}$ reportada para pacientes com glaucoma asiáticos ou caucasianos foi compatível com uma sintomatologia leve ${ }^{(7)}$.

Outro dado que pode ter contribuído para alta prevalência de sinais e sintomas de OSD neste estudo foi a preponderância do sexo feminino na amostra. No entanto, ainda assim, a prevalência de OSD reportada encontra-se bem acima da esperada para o sexo feminino de igual faixa etária na população geral ${ }^{(21)}$.

Não existe um consenso quanto aos critérios diagnósticos de OSD. Os testes e exames mais utilizados na avaliação da superfície ocular são: biomicroscopia, testes de Schirmer, avaliação da superfície ocular com corante rosa Bengala, fluoresceína ou lisamina verde, 
avaliação do TRFL, análise bioquímica do filme lacrimal e citologia de impressão da córnea e conjuntiva ${ }^{(22)}$. Para avaliar o impacto na qualidade de vida e permitir a padronização das queixas dos pacientes, são disponíveis diversos questionários, como o questionário desenvolvido por McMonnies(23), o questionário "Visual Function Questionnaire" (NFQ-25) desenvolvido pelo "National Eye Institute"(24) e o OSDI ${ }^{\circledR}$ elaborado pela companhia Allergan. O último se destaca por ter sido validado através de testes psicométricos e por ter sido aprovado pelo FDA (U.S. Food and Drug Administration) para uso em ensaios clínicos ${ }^{(12,25,26)}$

No presente estudo, foi realizado avaliação dos sinais e sintomas de OSD em pacientes com GPAA ou OHT. A avaliação dos sinais foi composta por avaliação estrutural da superfície ocular (por meio da biomicroscopia com e sem corantes vitais) e funcional (por meio do TRFL). Observou-se que 75\% dos pacientes apresentaram TRFL alterado, 20\% ceratite ponteada e 35\% apresentaram coloração positiva na córnea ou na conjuntiva pelo corante de rosa Bengala. No estudo de Leung et $\mathrm{al}^{\mid(3)}, 78 \%$ dos pacientes com GPAA ou OHT apresentaram TRFL alterado e $22 \%$ coloração conjuntival positiva, corroborando com os dados deste estudo.

Para melhor avaliação do impacto dos sintomas relacionados à OSD na qualidade de vida, foi utilizado o questionário OSDI ${ }^{\circledR}$. Este foi escolhido por ser um teste de rápida aplicação, além de permitir a geração de um índice mesmo que o paciente não tenha respondido todas as questões do teste. Estudos recentes também utilizaram o OSDI ${ }^{\circledR}$ para avaliar a prevalência de sintomas de OSD em pacientes com glaucoma, reportando uma prevalência de 44,4 a 59\%, dependendo dos valores de corte adotados $(3,8,10,27,28)$. No estudo de Rossi et al ${ }^{(8)}$, cerca de $44 \%$ dos pacientes em uso de colírios hipotensores oculares apresentaram sintomas de OSD. A classificação do OSDI ${ }^{\circledR}$ utilizada no estudo atual é a mesma usada por Rossi et al ${ }^{(8)}$. É possível que a maior prevalência de sintomas de OSD encontrada no estudo atual (67,5 \%) seja devido à maior duração do tratamento com hipotensores ocular tópicos desta amostra (9,8 anos), quando comparada à amostra do estudo de Rossi et al (2,5 anos).

De fato, o estudo atual mostrou que existe uma correlação positiva estatisticamente significativa $(p=0,01)$ entre a pontuação do $\mathrm{OSDI}^{\circledR}$ e o número de anos em terapia com hipotensores oculares tópicos. No entanto, o coeficiente de correlação foi de apenas 0,4. De acordo com a literatura, quando o coeficiente de correlação tomado em módulo é maior que 0,3 e menor ou igual a 0,6, a correlação deve ser considerada apenas moderada ${ }^{(29)}$. Tal correlação positiva também foi observada em estudo multicêntrico recente ${ }^{(7)}$. No entanto, por se tratar de estudo transversal, não podemos afirmar que os altos índices do OSDI ${ }^{\circledR}$ encontrados nesta amostra tenham sido provocados pelo tempo de tratamento. Seria necessário estudo prospectivo com análises seriadas das queixas avaliadas pelo mesmo questionário a fim de se confirmar esta hipótese.

Ao se comparar os grupos com menor e maior posologia, não foram encontradas diferenças estatisticamente significativas no que diz respeito ao OSDI ${ }^{\circledR}$, TRFL e teste com rosa Bengala. A longa duração do tratamento observada (9,4 anos no grupo 1 e 9,9 anos no grupo 2) pode ter induzido alterações importantes na superfície ocular mesmo em pacientes em monoterapia.

Neste estudo, blefarite foi diagnosticada em $42,5 \%$ dos pacientes. Blefarite inclui um grupo de afecções que acomete, de forma inflamatória, as pálpebras e/ou margens palpebrais e que pode envolver, secundariamente, a superfície ocular adjacente ${ }^{(30)}$. Apesar de ser uma alteração comum, a prevalência de blefarite em pacientes com glaucoma foi pouco estudada. Um estudo realizado por Uusilato et al. ${ }^{(31)}$ detectou blefarite em $60,1 \%$ dos participantes em uso crônico de uma formulação de análogo de prostaglandina com conservante. Os autores observaram que houve redução da frequência de blefarite e de outros sinais de OSD quando a terapia foi alterada para análogo de prostaglandina sem conservante ${ }^{(31)}$. De maneira semelhante, Henry et al. ${ }^{(32)}$ encontraram uma melhora na pontuação do OSDI ${ }^{\circledR}$ em 70\% dos pacientes quando a terapia foi alterada para uma formulação de análogo de prostaglandina sem conservante.

O reconhecimento e tratamento da OSD em pacientes glaucomatosos tem importância que vai desde melhora na qualidade de vida do pacienteaté melhora nos índices de confiabilidade do campo visual|(31-33). O tratamento da OSD em pacientes com glaucoma envolve tentar diminuir a toxicidade das medicações tópicas utilizadas e neste sentido, formulações sem BAK podem ser benéficas ${ }^{(4,31,32)}$.

Por último, cabe ressaltar que o estudo atual foi realizado em um centro que atende a população de baixa renda socioeconômica, com baixo nível de escolaridade e isto pode ter influenciado os resultados deste estudo, principalmente no que se refere ao questionário OSDI ${ }^{\circledR}$. Além disso, trata-se de um centro terciário de referência. Portanto, possivelmente reflete casos mais graves de glaucoma que necessitam de uso de múltiplas drogas para o controle da doença. Novos estudos avaliando o impacto da OSD em pacientes com glaucoma levando em consideração a gravidade do glaucoma necessitam ser conduzidos.

\section{CONCLUSÃO}

Pacientes com glaucoma primário de ângulo aberto (GPAA) ou hipertensão ocular $(\mathrm{OHT})$ em uso crônico de hipotensores oculares tópicos apresentam alta prevalência de sinais e sintomas de doença da superfície ocular (OSD). Existe correlação significativa entre a duração do tratamento e a gravidade dos sintomas de OSD.

\section{REFERÊNCIAS}

1. The definition and classification of dry eye disease: report of the Definition and Classification Subcommittee of the International Dry Eye WorkShop (2007). Ocul Surf. 2007;5(2):75-92

2. The epidemiology of dry eye disease: report of the epidemiology subcommittee of the International Dry Eye WorkShop (2007). Ocul Surf. 2007;5:93-107.

3. Leung EW, Medeiros FA, Weinreb RN. Prevalence of ocular surface diseases in glaucoma patients. J Glaucoma. 2008;17(5):350-355.

4. Stewart WC, Stewart JA, Nelson LA. Ocular surface disease in patients with ocular hypertension and glaucoma. Current Eye Research. 2011;36(5):391-398

5. O'Hare F, Ghosh S, Lamoureux E, Vajpayee RB, Crowston JG. Prevalence of signs and symptoms of ocular surface disease in individuals treated and not treated with glaucoma medication. Clin Experiment Ophthalmol. 2012 Mar 7. In press.

6. Mantelli F, Tranchina L, Lambiase A, Bonini S. Ocular surface damage by ophthalmic compounds. Curr Opin Allergy Clin Immunol. 2011;11(5):464-70.

7. Garcia-Feijoo J, Sampaolesi JR. A multicenter evaluation of ocular surface disease prevalence in patients with glaucoma. Clin Ophthalmol. 2012;6:441-6.

8. Rossi GC, Tinelli C, Pasinetti GM, Milano G, Bianchi PE. Dry eye syndrome-related quality of life in glaucoma patients. Eur J Ophthalmol. 2009;19(4):572-9.

9. Van Went C, Brasnu E, Hamard P, Baudouin C, Labbé A. [The influence of ocular surface diseases in the management of glaucoma]. J Fr Ophtalmol. 2011;34(4):230

10. Fechtner RD, Godfrey DG, Budenz D, Stewart JA, Stewart WC, Jasek MC. Prevalence of ocular surface complaints in patients with glaucoma using topical intraocular pressure-lowering medications. Cornea. 2010;29(6):618-21.

11. Baudouin C. Mechanisms of failure in glaucoma filtering surgery: a consequence of antiglaucomatous drugs? Int J Clin Pharmacol Res. 1996;16(1):29-41.

12. Shiffman RM, Christianson MD, Jacobsen G, Hirsch JD, Reis BL. Reliability and validity of the ocular surface disease index. Arch Ophthalmol 2000; 118: 615-21.

13. Walt J. Ocular Surface Disease Index (OSDI) Administration and Scoring Manual. Irvine, CA: Allergan, Inc; 2004

14. Van Bijsterveld OP. Diagnostic tests in the Sicca syndrome. Arch Ophthalmol. 1969; 82(1):10-14.

15. Baffa LP, Ricardo JRS, Dias AC, Módulo CM, Braz AM, Paula JS, et al. Tear film and ocular surface alterations in chronic users of antiglaucoma medications. Arq Bras Oftalmologia. 2008;71(1):18-21.

16. Tsai JH, Derby E, Holland EJ, Khatana AK. Incidence and prevalence of glaucoma in severe ocular surface disease. Cornea. 2006;25(5):530-532.

17. Servat JJ, Bernardino CR. Effects of common topical antiglaucoma medications on the ocular surface, eyelids and periorbital tissue. Drugs Aging. 2011;28(4):267-82.

18. Brandt JD, Wittpenn JR, Katz LJ, Steinmann WN, Spaeth GL. Conjunctival impression cytology in patients with glaucoma using long-term topical medication. Am J Ophthalmol. 1991;112(3):297-301

19. Noecker R, Miller KV. Benzalkonium chloride in glaucoma medications. Ocul Surf. 2011;9(3):159-62.

20. Pisella, PJ, Pouliquen, P, Baudouin C. Prevalence of ocular symptoms and signs with 
preserved and preservative free glaucoma medication. Br J Ophthalmol. 2002; (86):418-423.

21. Schaumberg DA, Sullivan DA, Buring JE,Dana MR. Prevalence of dry eye syndrome among US women. Am J Ophthalmol. 2003 Aug;136(2):318-26.

22. Nishiwaki-Dantas MC. Atualização em olho seco. Arq Bras Oftalmol. 2000; 62(1):101-5.

23. McMonnies CW. Key questions in dry eye history. J Am Optom Assoc. 1986; 57(7):512-7.

24. Mangione CM, Lee PP, Gutierrez PR, Spritzer K, Bwerry S, Hay RD; National Eye Institute Visual Function Questionnaire Field Test Investigators. Development of the 25-item National Eye Institute Visual Function Questionnaire. Arch Ophthalmol. 2001;119(7):1050-8.

25. Johnson ME, Murphy PJ. Measurement of ocular surface irritation on a linear interval scale with the ocular comfort index. Invest Ophthalmol Vis Sci. 2007:48(10):4451-8.

26. Prigol AM, Tenório MB, Matschinske R, Gehlen ML, Share T. [Translation and validation of ocular surface disease index to portuguese]. Arq Bras Oftalmol. 2012; 75(1):24-8.
27. Valente C, lester M, Corsi E, Rolando M. Symptoms and signs of tear film dysfunction in glaucomatous patients.J Ocul Pharmacol Ther. 2011;27(3):281-5.

28. Skalicky SE, Goldberg I, McCluskey P. Ocular Surface Disease and Quality of Life in Patients With Glaucoma. Am J Ophthalmol. 2012;153(1):1-9

29. Taylor R. Interpretation of the correlation coefficient a basic review. J Diagn Med Sonog. 1990;1:35-9

30. Bernardes TF, Bonfioli AA. Blepharitis. Semin Ophthalmol. 2010;25(3):79-83.

31. Uusitalo H, Chen E, Pfeiffer N, Brignole-Baudouin F, Kaarniranta K, Leino M, et al. Switching from a preserved to a preservative-prostaglandin preparation in topical glaucoma medication. Acta Ophthalmol. 2010;88(3):329-36

32. Henry JC, Peace JH, Stewart JA, Stewart WC. Efficacy, safety, and improved tolerability of travoprost BAK-free ophthalmic solution compared with prior prostaglandin therapy. Clin Ophthalmol. 2008; 2(3):613-21.

33. Yenice $O$, Temel A, Orüm $O$. The effect of artificial tear administration on visual field testing in patients with glaucoma and dry eye. Eye (Lond). 2007;21(2):214-7.

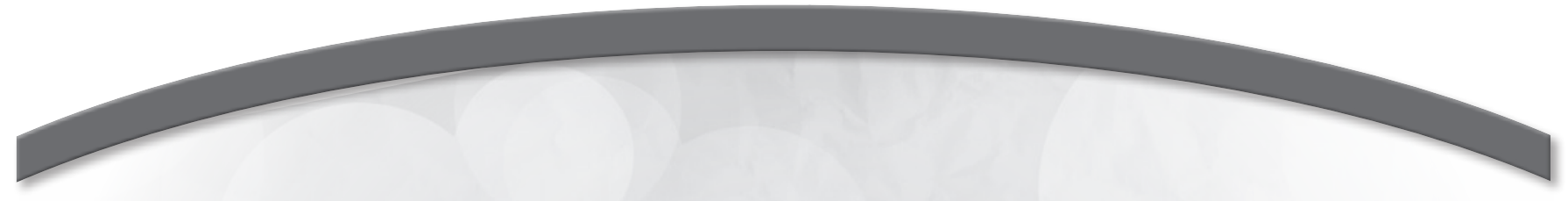

\title{
XX Congresso Norte-Nordeste de Oftalmologia
}

\author{
27 a 29 de março de 2014 \\ Fortaleza (CE)
}

Informações:

E-mail: secretaria@snno.com.br

Site: http://www.snno.com.br/

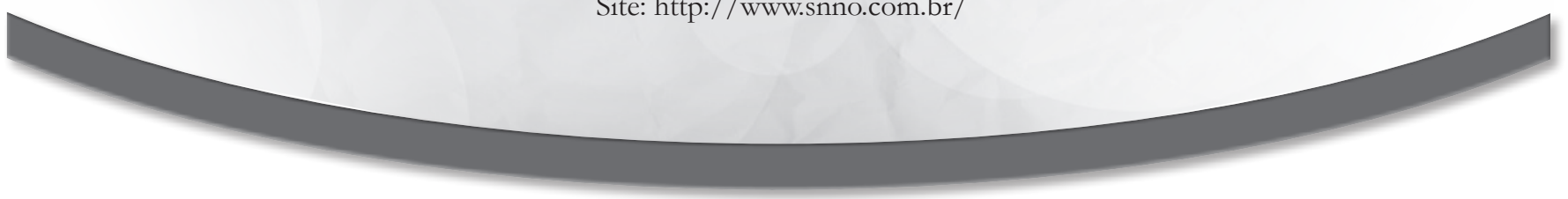

